\title{
Reinforced Weak Convergence of Stochastic Processes
}

\author{
Michael Drmota*and Jean-François Marckert ${ }^{\dagger}$
}

November 30, 2004

\begin{abstract}
We consider a sequence of stochastic processes $X_{n}$ on $C[0,1]$ converging weakly to $X$ and call it polynomially convergent, if $\mathbf{E} F\left(X_{n}\right) \rightarrow \mathbf{E} F(X)$ for continuous functionals $F$ of polynomial growth. We present a sufficient moment conditions on $X_{n}$ for polynomial convergence and provide several examples, e.g. discrete excursions and depth first path associated to Galton-Watson trees. This concept leads to a new approach to moments of functionals of rooted trees such as height and path length.
\end{abstract}

Keywords: weak convergence, excursions, functionals of trees.

\section{Introduction}

Let $X_{n}$ and $X$ denote stochastic processes on $C[0,1]$. Then weak convergence of $X_{n}$ to $X$ means that for every continuous and bounded functional $F: C[0,1] \rightarrow \mathbf{R}$, we have

$$
\mathbf{E} F\left(X_{n}\right) \rightarrow \mathbf{E} F(X) \quad(n \rightarrow \infty) .
$$

The topology on $C[0,1]$ is induced by the norm $\|\cdot\|_{\infty}$. The purpose of this paper is to show that, under natural moments assumptions, this property can be extended to a wider class of functionals which need not to be bounded anymore.

For this purpose we introduce a notion of reinforced weak convergence.

Definition Let $X_{n}$ and $X$ denote continuous stochastic processes on $C[0,1]$. We say that $X_{n}$ converges polynomially to $X$ if

$$
\mathbf{E} F\left(X_{n}\right) \rightarrow \mathbf{E} F(X) \quad(n \rightarrow \infty)
$$

for all continuous functionals $F: C[0,1] \rightarrow \mathbf{R}$ satisfying

$$
|F(f)| \leq C\left(1+\|f\|_{\infty}\right)^{k}
$$

for some constants $C, k>0$.

Remark 1 Examples for functionals $F$ satisfying (2) are maximum and integrals. Hence, if $X_{n}$ is polynomially convergent to $X$ we have, for any $r>0$ and for any Borel set $I \subset[0,1]$

$$
\mathbf{E} \max _{t \in I}\left|X_{n}(t)\right|^{r} \rightarrow \mathbf{E} \max _{t \in I}|X(t)|^{r}
$$

and

$$
\mathbf{E} \int_{I} X_{n}(t)^{r} d t \rightarrow \mathbf{E} \int_{I} X(t)^{r} d t .
$$

${ }^{*}$ Institute of Discrete Mathematics and Geometry, Vienna University of Technology, Wiedner Hauptstrasse 8-10, A-1040 Vienna, Austria.

Corresponding Author

${ }^{\dagger}$ LAMA, Université de Versailles-St. Quentin, Bâtiment Fermat, 45 Avenue des Etats-Unis, 78035 Versailles, France 
Remark 2 Note that polynomial convergence is equivalent to

$$
\sup _{n} \mathbf{E}\left(\left\|X_{n}\right\|_{\infty}^{k}\right)<\infty \quad(\text { for all } k>0)
$$

if $X_{n}$ converges weakly to $X$. This follows almost directly from Billingsley (1995, p. 338) (see also Lemma 1 and Lemma 2).

We first state a necessary condition for polynomial convergence.

Theorem 1 Suppose that $X_{n}$ and $X$ are stochastic processes on $C[0,1]$ such that $X_{n}$ converges weakly to $X$. Further assume that the following two conditions are satisfied

1. There exists $t_{0} \in[0,1]$ such that for all integers $k \geq 0$

$$
\sup _{n} \mathbf{E}\left|X_{n}\left(t_{0}\right)\right|^{k}<\infty
$$

2. There exists a sequence $\left(\alpha_{d}\right)_{d \geq 1}$ of positive real numbers with $\alpha_{d}>1$ for infinitely many $d$ such that for all integers $d>0$

$$
\mathbf{E}\left|X_{n}(t)-X_{n}(s)\right|^{d} \leq C_{d}|t-s|^{\alpha_{d}} \quad \text { for all } s, t \in[0,1]
$$

for some constant $C_{d}>0$.

Then $X_{n}$ converges polynomially to $X$.

Remark 3 Note that (4) and (5) imply tightness of $X_{n}$ by applying Kolmogorov's criterion (see Revuz and

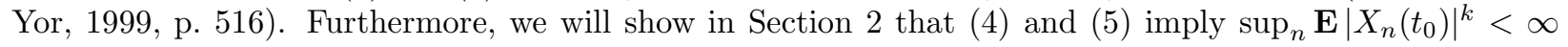
for all fixed $t_{0} \in[0,1]$ and integers $k \geq 0$. Consequently it follows from Cauchy-Schwarz's inequality and Lemma 1 that

$$
\mathbf{E}\left(X\left(t_{1}\right)^{k_{1}} X\left(t_{2}\right)^{k_{2}} \cdots X\left(t_{m}\right)^{k_{m}}\right)
$$

exists and

$$
\mathbf{E}\left(X_{n}\left(t_{1}\right)^{k_{1}} X_{n}\left(t_{2}\right)^{k_{2}} \cdots X_{n}\left(t_{m}\right)^{k_{m}}\right) \rightarrow \mathbf{E}\left(X\left(t_{1}\right)^{k_{1}} X\left(t_{2}\right)^{k_{2}} \cdots X\left(t_{m}\right)^{k_{m}}\right) \quad(n \rightarrow \infty)
$$

for all $m \geq 1,0 \leq t_{1}<t_{2}<\cdots<t_{m} \leq 1$ and integers $k_{j} \geq 0, j=1,2, \ldots, m$. This property may be interpreted as a moment version of finite dimensional convergence.

Thus, the conditions of Theorem 1 are (more or less) strengthened versions of tightness and finite dimensional convergence - two properties which are equivalent to weak convergence.

The paper is organized as follows: The proof of Theorem 1 is presented in Section 2. In Section 3 we show that polynomial convergence may be interpreted as a property lying between weak and strong convergence. Finally Section 4 is devoted to applications of Theorem 1 concerning trees and excursions. For example it is shown that discrete excursions (related to random walks) converge polynomially to Brownian excursion (Theorem 4).

\section{Proof of Theorem 1}

In the Introduction we have already mentioned that it is sufficient to prove (3). In order to be more precise we first state a result of Billingsley (1995, p. 338) which will be used then to prove this property.

Lemma 1 Suppose that the sequence of random variables $Y_{n}$ converges weakly to $Y$ and that $\sup _{n} \mathbf{E}\left|Y_{n}\right|^{r+\varepsilon}<$ $\infty$ for some positive integer $r$ and some $\varepsilon>0$. Then $\mathbf{E}|Y|^{r}<\infty$ and $\mathbf{E} Y_{n}^{r} \rightarrow \mathbf{E} Y^{r}$ (as $\left.n \rightarrow \infty\right)$. 
Lemma 2 Suppose that $X_{n}$ converges weakly to $X$ and (3) is satisfied. Then $X_{n}$ converges polynomially to $X$.

Proof. Without loss of generality we may assume that $F(f) \leq C\left(1+\|f\|_{\infty}\right)^{k}$ for some positive integer $k$. Then for $\varepsilon=1 / k$ we have

$$
\left|F\left(X_{n}\right)\right|^{1+\varepsilon} \leq C^{1+1 / k}\left(1+\left\|X_{n}\right\|\right)^{k+1} .
$$

and consequently

$$
\sup _{n} \mathbf{E}\left(\left|F\left(X_{n}\right)\right|^{1+\varepsilon}\right)<\infty .
$$

Thus, we can apply Lemma 1 with $Y_{n}=F\left(X_{n}\right)$ and (1) follows.

In order to verify (3) we will mainly make use of the following property.

Lemma 3 Suppose that $X$ and $X_{n}$ are stochastic processes satisfying the assumptions of Theorem 1. Then for every integer $d \geq 0$ for which $\alpha_{d}>1$ there exists a constant $K>0$ such that for $\varepsilon>0,0<\delta<1$, and $n \geq 0$

$$
\operatorname{Pr}\left\{\sup _{|s-t| \leq \delta}\left|X_{n}(s)-X_{n}(t)\right| \geq \varepsilon\right\} \leq K \frac{\delta^{\alpha_{d}-1}}{\varepsilon^{d}}
$$

and consequently for every fixed $k \leq d-1$ there is a constant $K^{\prime}$ such that for $0<\delta<1$ and $n \geq 0$

$$
\mathbf{E}\left(\sup _{|s-t| \leq \delta}\left|X_{n}(s)-X_{n}(t)\right|^{k}\right) \leq K^{\prime} \delta^{k \frac{\alpha_{d}-1}{d}} .
$$

Proof. First (6) follows from (5) by using the methods of Billingsley (1968, pp. 95).

Next set

$$
Z_{n}:=\sup _{|s-t| \leq \delta}\left|X_{n}(s)-X_{n}(t)\right|
$$

and $\bar{F}_{n}(y)=\operatorname{Pr}\left\{Z_{n} \geq y\right\}$. Hence, with $A=\delta^{\frac{\alpha_{d}-1}{d}}$ we obtain

$$
\begin{aligned}
\mathbf{E} Z_{n}^{k} & =-\int_{0}^{\infty} y^{k} d \bar{F}_{n}(y) \\
& =k \int_{0}^{\infty} y^{k-1} \bar{F}_{n}(y) d y \\
& =k \int_{0}^{A} y^{k-1} \bar{F}_{n}(y) d y+k \int_{A}^{\infty} y^{k-1} \bar{F}_{n}(y) d y \\
& \leq k \int_{0}^{A} y^{k-1} d y+K k \delta^{\alpha_{d}-1} \int_{A}^{\infty} y^{k-1-d} d y \\
& \leq A^{k}+K \frac{k}{d-k} A^{k-d} \delta^{\alpha_{d}-1} \\
& \leq K^{\prime} \delta^{k \frac{\alpha_{d}-1}{d}} .
\end{aligned}
$$

It is now easy to prove (3). First of all it follows from

$$
\mathbf{E}\left|X_{n}(t)\right|^{k} \leq 2^{k} \mathbf{E}\left|X_{n}(t)-X_{n}\left(t_{0}\right)\right|^{k}+2^{k} \mathbf{E}\left|X_{n}\left(t_{0}\right)\right|^{k}
$$

that

$$
\sup _{n} \mathbf{E}\left|X_{n}(t)\right|^{k}<\infty
$$


for all fixed $t \in[0,1]$ and integers $k>0$. (We will use this property for $t=\frac{1}{2}$.)

Next we apply (7) for $\delta=1 / 2$ and get

$$
\begin{aligned}
\mathbf{E}\left(\sup _{0 \leq t \leq 1}\left|X_{n}(t)\right|^{k}\right) & \leq 2^{k} \mathbf{E}\left|X_{n}(1 / 2)\right|^{k} \\
& +2^{k} \mathbf{E}\left(\sup _{|s-t| \leq 1 / 2}\left|X_{n}(s)-X_{n}(t)\right|^{k}\right) \\
& =\mathcal{O}(1) .
\end{aligned}
$$

Thus, (3) is satisfied and Theorem 1 follows.

\section{Weak convergence and "strong approximation"}

It is always a natural question to prove a better convergence than only weak convergence when one studies the convergence of stochastic processes to their limit. In this section we want to present a result on "strong approximation" and show that polynomial convergence is implied by it.

Consider a sequence $\left(Y_{n}\right)_{n>1}$ of i.i.d. random variables such that $\mathbf{E}\left(Y_{1}\right)=0$ and $0<\operatorname{Var}\left(Y_{1}\right)=\sigma^{2}<+\infty$. We denote by $S_{n}$ the partial sum $S_{n}=Y_{1}+\cdots+Y_{n}$ and define the sequence of processes $X_{n}$ by:

$$
X_{n}(t)=\frac{1}{\sigma \sqrt{n}} S_{\lfloor n t\rfloor}+(n t-\lfloor n t\rfloor) \frac{1}{\sigma \sqrt{n}} Y_{\lfloor n t\rfloor+1}, \quad \text { for } 0 \leq t \leq 1 .
$$

A very classical weak convergence theorem due to Donsker (1951) (see Billingsley, 1968, p. 68) says that $X_{n}$ converges weakly to Brownian motion of duration 1; this is shown by proving convergence of finite dimensional distribution and providing a tightness property.

A stronger result is obtained by Komlós, Major and Tusnády (see Csörgő and Horváth, 1993, for a collection of such results).

Theorem 2 (KMT) Suppose that $\mathbf{E}\left(e^{u Y_{1}}\right)<+\infty$ for $u$ in a neighborhood of zero. Then we can define a sequence of Brownian motions $\left\{W_{n}(t), 0 \leq t \leq 1\right\}$ such that

$$
\operatorname{Pr}\left(\left\|X_{n}-W_{n}\right\|_{\infty} \geq \frac{x+c_{1} \log n}{\sqrt{n}}\right) \leq c_{2} e^{-c_{3} x}, \quad \text { for any } x \geq 0
$$

where $c_{1}, c_{2}, c_{3}$ are positive constants just depending of the distribution of $Y_{1}$.

The proof uses coupling arguments, especially the variables $Y_{n}$ are built as functionals of the Brownian path and are, thus, defined on the same probability space.

There exists a similar theorem for the "strong approximation" of the discrete Poisson bridge (random walk with Poisson increments conditioned by the event $\left\{S_{n}=0\right\}$ ) to the Brownian bridge (see Csörgö and Horváth, 1993, for applications in the study of random Cayley trees and parking functions).

We now indicate that Theorem 2 implies polynomial convergence. Indeed, according to Lemma 2 it is sufficient to prove

$$
\sup _{n} \mathbf{E}\left(\left\|X_{n}\right\|_{\infty}^{k}\right)<+\infty .
$$

for all $k>0$. For this purpose we use the relation

$$
\mathbf{E}\left(\left\|X_{n}\right\|_{\infty}^{k}\right) \leq 2^{k} \mathbf{E}\left(\left\|X_{n}-W_{n}\right\|_{\infty}^{k}\right)+2^{k} \mathbf{E}\left(\left\|W_{n}\right\|_{\infty}^{k}\right) .
$$

Since the maximum of the Brownian motion has for law the absolute value of a centered Gaussian r.v. with 
variance 1, we just have to deal with the first term on the right hand side:

$$
\begin{aligned}
\mathbf{E}\left(\left\|X_{n}-W_{n}\right\|_{\infty}^{k}\right) & =\int_{\mathbb{R}^{+}} \operatorname{Pr}\left(\left\|X_{n}-W_{n}\right\|_{\infty}^{k} \geq x\right) d x \\
& \leq 1+\int_{1}^{+\infty} \operatorname{Pr}\left(\left\|X_{n}-W_{n}\right\|_{\infty}^{k} \geq x\right) d x \\
& \leq 1+\int_{0}^{+\infty} c_{2} e^{-c_{3} t} \frac{k}{\sqrt{n}}\left(\frac{c_{1} \log n+t}{\sqrt{n}}\right)^{k-1} d t
\end{aligned}
$$

where we have used the change of variables $x=\left(\left(c_{1} \log n+t\right) / \sqrt{n}\right)^{k}$. This is bounded uniformly on $n$.

Thus, polynomial convergence lies between the standard weak convergence and strong approximation (in the sense of Theorem 2).

We also want to mention that there are variations of Theorem 2 (e.g. Theorem 1.11 of Csörgö and L. Horváth, 1993) which can be used to prove polynomial convergence of $X_{n}$, too, even if we only know that all moments of $Y_{1}$ exist. In this case it is also possible to check polynomial convergence of $X_{n}$ via Theorem 1. (4) is trivial for $t_{0}=0$ and (5) can be checked with help of a result of Fuk \& Nagaev (1971).

\section{Applications to Trees and Excursions}

It is well known that a lot of processes associated to simple trees by traversal algorithms (depth first search, breadth first search...) converges weakly to the Brownian excursion (or to Brownian excursion local time) see for example Aldous (1991), Drmota and Gittenberger (2004), and Marckert and Mokkadem (2003) It was one motivation of this paper to show that the convergence of these processes to Brownian excursion is not only weak, but polynomial.

Section 4 is organized as follows: in Subsection 4.1 we show that Theorem 1 applies to the contour of simple trees (that is the process of the height of leaves). As corollaries of Subsection 4.1 we prove polynomial convergence of two important classes of processes to Brownian excursion by applying concentration results of Marckert and Mokkadem (2003): in Subsection 4.2 we show that discrete excursions (under some usual conditions) converge polynomially to Brownian excursion, and in Subsection 4.3 we prove that the depth first walk associated to simple trees enjoys the same property.

\subsection{The Contour of Trees}

In this section we show that Theorem 1 applies to the contour of trees related to (critical) Galton-Watson branching processes $\left(Z_{k}\right)_{k \geq 0}$ conditioned on the size.

Let $\xi$ be a non-negative integer valued random variable with $\mathbf{E} \xi=1,0<\operatorname{Var} \xi=\sigma^{2}<\infty$, and suppose that there is $\alpha>0$ such that $\mathbf{E} e^{\alpha \xi}<\infty$. $\left(Z_{k}\right)_{k \geq 0}$ is now given by $Z_{0}=1$, and for $k \geq 1$,

$$
Z_{k}=\sum_{j=1}^{Z_{k-1}} \xi_{j}^{(k)},
$$

where the $\left(\xi_{j}^{(k)}\right)_{k, j}$ are i.i.d. random variables distributed as $\xi$.

It is well known that Galton-Watson branching processes can be represented by ordered rooted trees $T .{ }^{1}$ We will denote $\nu(T)$ the probability that $T$ occurs. The generating function $y(x)=\sum_{n \geq 1} y_{n} x^{n}$ of the numbers

$$
y_{n}=\sum_{|T|=n} \nu(T)
$$

\footnotetext{
${ }^{1}$ For a critical branching process the probability that $T$ is finite equals 1 .
} 
satisfies the functional equation

$$
y(x)=x \varphi(y(x)),
$$

where $\varphi(t)=\mathbf{E} t^{\xi}=\sum \varphi_{i} t^{i}$ with $\varphi_{i}=\operatorname{Pr}\{\xi=i\}$. If $\mathcal{T}_{n}$ denotes the set of rooted trees $T$ such that $|T|=n$ then

$$
\nu_{n}(T):=\frac{\nu(T)}{y_{n}}
$$

is a probability distribution on $\mathcal{T}_{n}$ which we will use in the sequel. Note that

$$
y_{n}=\frac{d}{\sqrt{2 \pi} \sigma} n^{-3 / 2}+O\left(n^{-5 / 2}\right) \quad(n \equiv 1 \bmod d),
$$

where $d=\operatorname{gcd}\left\{i>0: \varphi_{i}>0\right\}$.

Let $T$ denote a finite tree of size $n$ appearing in such a branching process. There is a natural left to right order of the descendents and consequently a natural left to right numeration of the leaves $L(T)$ (in consistence with the depth first traversal). It is well known that the expected number of leaves in trees of size $n$ is given by

$$
\sum_{|T|=n}|L(T)| \nu_{n}(T)=\varphi_{0} n+\mathcal{O}(1)
$$

and that there is a central limit law with variance of order $n$. Thus, $|L(T)| / n$ is concentrated around $\varphi_{0}$ for trees $T \in \mathcal{T}_{n}$.

We want to get some informations on the contour $h_{n}^{(0)}$ of these trees $\mathcal{T}_{n}$ as $n$ tends to infinity (for the reader's convenience we use the notation of Marckert and Mokkadem, 2003). For an integer $j \geq 1$ let $h_{n}^{(0)}(j)$ denote the height of the $j$-th leaf (in the natural left to right order). In order to get a process of continuous functions we use linear interpolation between these discrete values. We also set $h_{n}^{(0)}(0)=0$ and $h_{n}^{(0)}(k)=0$ if $k$ is larger than the number of leaves. The normalized contour is then given by

$$
\tilde{e}_{n}(t):=\frac{1}{\sqrt{n}} h_{n}^{(0)}\left(n t \varphi_{0}\right)
$$

It has been shown by Gittenberger (1999) (see also Marckert and Mokkadem, 2003, for probabilistic approach) that

$$
\tilde{e}_{n} \stackrel{\text { weakly }}{\longrightarrow} \frac{2}{\sigma} e
$$

where $\{e(t), 0 \leq t \leq 1\}$, denotes the Brownian excursion of duration 1 .

We want to show that this weak convergence property can be strengthened to polynomial convergence.

Theorem 3 (Polynomial convergence of the contour of trees) The sequence of processes $\tilde{e}_{n}$ converges polynomially to $\frac{2}{\sigma} e$.

For this purpose we apply Theorem 1, i.e. we have to prove (4) and (5). (4) is trivially satisfied since $X_{n}(0)=0$.

In order to show (5) we use the following estimate which is already contained in Gittenberger (1999), compare also with Drmota and Gittenberger (2004).

Lemma 4 There exist constants $C, D>0$ such that for all $s, t \in[0,1]$ and $\epsilon>0$,

$$
\operatorname{Pr}\left\{\left|\tilde{e}_{n}(s)-\tilde{e}_{n}(t)\right| \geq \varepsilon\right\} \leq \frac{C}{\varepsilon^{4}|s-t|} \exp \left(-D \frac{\varepsilon}{\sqrt{|s-t|}}\right)
$$

Now (5) follows from the following property. 
Lemma 5 Suppose that a sequence of processes $X_{n}$ on $C[0,1]$ satisfies

$$
\operatorname{Pr}\left\{\left|X_{n}(s)-X_{n}(t)\right| \geq \varepsilon\right\} \leq \frac{C}{\varepsilon^{\eta_{1}}|s-t|^{\eta_{2}}} \exp \left(-D \frac{\varepsilon}{\sqrt{|s-t|}}\right) \quad \text { for all } s, t \in[0,1]
$$

for some positive constants $C, D, \eta_{1}, \eta_{2}$. Then for every integer $d>0$ there exists a constant $C_{d}>0$ such that

$$
\mathbf{E}\left|X_{n}(t)-X_{n}(s)\right|^{2 d} \leq C_{d}|t-s|^{d} \quad \text { for all } s, t \in[0,1] .
$$

Proof. In order to simplify our notation we set

$$
Z_{n}:=\left|X_{n}(s)-X_{n}(t)\right|
$$

and $\bar{F}_{n}(y)=\operatorname{Pr}\left\{Z_{n}>y\right\}$. Note that for every $m>0, e^{-x}=\mathcal{O}\left(x^{-m}\right)$ for $x \geq 1$. Thus, taking $m=d+2 \eta_{2}$ (8) gives

$$
\begin{aligned}
\bar{F}_{n}(y) & =\mathcal{O}\left(\frac{1}{y^{\eta_{1}}|s-t|^{\eta_{2}}} \exp \left(-D \frac{y}{\sqrt{|s-t|}}\right)\right) \\
& =\mathcal{O}\left(\frac{1}{y^{\eta_{1}}|s-t|^{\eta_{2}}} \frac{|s-t|^{\eta_{2}+d / 2}}{y^{d+2 \eta_{2}}}\right) \\
& =\mathcal{O}\left(\frac{|s-t|^{d / 2}}{y^{d+\eta_{1}+2 \eta_{2}}}\right)
\end{aligned}
$$

for $y \geq \sqrt{|s-t|}$. Hence

$$
\begin{aligned}
\mathbf{E} Z_{n}^{d} & =-\int_{0}^{\infty} y^{d} d \bar{F}_{n}(y) \\
& =d \int_{0}^{\infty} y^{d-1} \bar{F}_{n}(y) d y \\
& =d \int_{0}^{\sqrt{|s-t|}} y^{d-1} \bar{F}_{n}(y) d y+d \int_{\sqrt{|s-t|}}^{\infty} y^{d-1} \bar{F}_{n}(y) d y \\
& =d \int_{0}^{\sqrt{|s-t|}} y^{d-1} d y+\mathcal{O}\left(d \int_{\sqrt{|s-t|}}^{\infty} \frac{|s-t|^{d}}{y^{1+\eta_{1}+2 \eta_{2}}} d y\right) \\
& =\mathcal{O}\left(|s-t|^{d / 2}\right)
\end{aligned}
$$

and the lemma is proved.

\subsection{Polynomial convergence of discrete excursions to Brownian excursion}

In this part, we show that under some natural conditions, discrete excursion converges polynomially to Brownian excursion.

Let $\left(S_{n}\right)_{n \geq 0}$ denote a random walk with i.i.d. increments $\left(\lambda_{i}\right)_{i \geq 1}$ with law given by $\left(\tilde{\varphi}_{i}\right)_{i \geq-1}$, that is:

$$
\operatorname{Pr}\left(\lambda_{1}=k\right)=\tilde{\varphi}_{k}=\varphi_{k+1},
$$

and

$$
S_{0}=0, \text { and } S_{k}=S_{k-1}+\lambda_{k}, \text { for } k \geq 1 .
$$

We will study the convergence of the process $\left(S_{k}\right)_{k=0, \ldots, n}$ conditioned by the event $\left\{S_{0}=0, S_{1} \geq\right.$ $\left.0, \cdots, S_{n-1}=0, S_{n}=-1\right\}$ to Brownian excursion. The convergence of the process $S_{n}$ (suitably normalized) 
to $e$ is well known. But, as far as we know, there is no strong approximation theorem of $S_{n}$ to $e$. (for example, Komlós, Major and Tusnády type theorem) that gives the speed of convergence. The aim of this subsection is to prove the following Theorem:

Theorem 4 (Polynomial convergence of discrete excursion) The sequence of processes

$$
X_{n}(t)=\frac{S_{n}(n t)}{\sqrt{n}} \quad(0 \leq t \leq 1)
$$

converges polynomially to $X=\sigma e$.

Note that $\lambda_{1}$ is distributed as $\xi_{1}-1$, where $\xi_{1}$ is the offspring distribution defined in the beginning of Subsection 4.1; hence, the only negative increment of $S_{n}$ are -1 and $S_{n}$ appears to be the Lukasiewiecz path (called depth first queue process (DFQP) in ) associated to the family representation of $\tau$ conditioned by $|\tau|=n$.

In order to prove this Theorem, we will use the strong relation between $S_{n}$ and $h_{n}^{(0)}$ given in Marckert and Mokkadem (2003) and Theorem 3. First let us state a corollary of Theorems 3 and 4 of Marckert and Mokkadem (2003):

Lemma 6 For any positive $\nu$, there exists two constants $\gamma>0$ and $N>0$ such that

$$
\forall n \geq N, \quad \operatorname{Pr}\left(\left\|S_{n}(n \cdot)-\frac{\sigma^{2}}{2} h_{n}^{(0)}\left(n \varphi_{0}\right)\right\|_{\infty} \geq n^{1 / 4+\nu}\right) \leq \exp \left(-\gamma n^{\nu}\right) .
$$

Proof of Theorem 4. Let $e_{n}^{\prime}$ denote the process defined by

$$
e_{n}^{\prime}(t):=\frac{1}{\sqrt{n}} S_{n t}, \text { for } t \in[0,1]
$$

and by linear interpolation between the integer values of $n t$. Then Lemma 6 says that for any positive $\nu$, there exists a $\gamma>0$ such that, if $n$ is sufficiently large,

$$
\operatorname{Pr}\left(\left\|e_{n}^{\prime}-\frac{\sigma^{2}}{2} \tilde{e}_{n}\right\|_{\infty} \geq n^{-1 / 4+\nu}\right) \leq \exp \left(-\gamma n^{\nu}\right)
$$

We already know that $\tilde{e}_{n}$ is polynomially convergent. In order to prove a corresponding property for $e_{n}^{\prime}$ we have to show that

$$
\sup _{n} \mathbf{E}\left(\left\|e_{n}^{\prime}\right\|_{\infty}^{k}\right)<\infty
$$

Since $\mathbf{E}\left\|\frac{\sigma^{2}}{2} \tilde{e}_{n}\right\|_{\infty}^{k}$ is bounded over $n$ and

$$
\mathbf{E}\left(\left\|e_{n}^{\prime}\right\|_{\infty}^{k}\right) \leq 2^{k} \mathbf{E}\left(\left\|e_{n}^{\prime}-\frac{\sigma^{2}}{2} \tilde{e}_{n}\right\|_{\infty}^{k}\right)+2^{k} \mathbf{E}\left(\left\|\frac{\sigma^{2}}{2} \tilde{e}_{n}\right\|_{\infty}^{k}\right)
$$

it is (surely) sufficient to show that

$$
\mathbf{E}\left(\left\|\frac{\sigma^{2}}{2} \tilde{e}_{n}-e_{n}^{\prime}\right\|_{\infty}^{k}\right) \rightarrow 0
$$

for all $k>0$. By construction, we have $\tilde{e_{n}} \leq \sqrt{n}$ and $e_{n}^{\prime} \leq \sqrt{n}$ (this comes from the constraints that the height of a leave in a tree is smaller that the size of the tree, and that $S_{n}$ is also smaller than $n$ since $S_{n}=-1$ and that the only negative increments are -1 ). Thus, one gets

$$
\mathbf{E}\left(\left\|\frac{\sigma^{2}}{2} \tilde{e}_{n}-e_{n}^{\prime}\right\|_{\infty}^{k}\right) \leq \operatorname{Pr}\left(\left\|\frac{\sigma^{2}}{2} \tilde{e}_{n}-e_{n}^{\prime}\right\|_{\infty}^{k} \geq n^{k(-1 / 4+\nu)}\right)\left(\frac{\sigma^{2}}{2}+1\right) n^{k / 2}+n^{k(-1 / 4+\nu)}
$$

Taking $0<\nu<1 / 4,(10)$ shows that this last quantity goes to 0 when $n$ goes to $+\infty$.

Remark 4 Polynomial convergence of the Lukasiewiecz path to Brownian excursion in the case of Poisson discrete excursion is used in Spencer (1997) to identify the Wright constants. 


\subsection{Convergence of the depth first walk associated to simple trees}

We define $V_{n}$ the depth first walk (DFW) associated to $\tau$ (under the same settings that in the preceding subsection, and with linear interpolation between the integer values): $V_{n}$ is a $2 n-2$ length walk that can be considered as a walk around the tree (see Aldous, 1991, or Marckert and Mokkadem, 2003, and Figure 1).
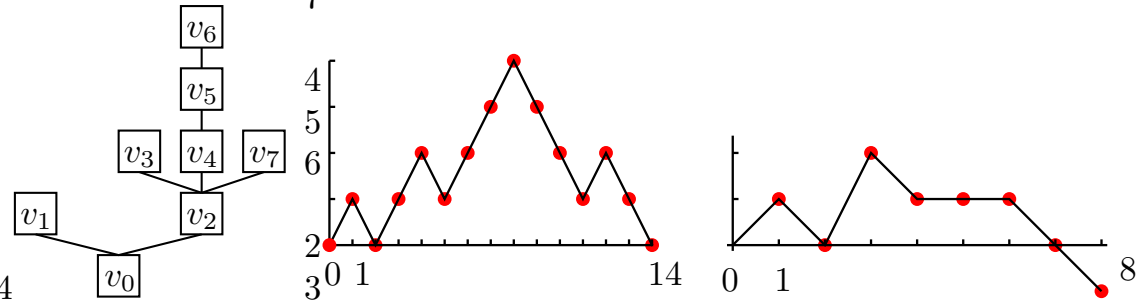

Figure 1: A tree and its associated DFW, DFQP.

According to Marckert and Mokkadem (2003, Theorem 2), we know that for any positive $\nu$ and sufficiently large $n$

$$
\operatorname{Pr}\left(\left\|\tilde{e}_{n}-\frac{V_{n}((2 n-2) \cdot)}{\sqrt{n}}\right\|_{\infty} \geq n^{-1 / 4+\nu}\right) \leq \exp \left(-\gamma n^{\nu}\right) .
$$

Following the same arguments that in the previous subsection, we obtain:

Theorem 5 (Polynomial convergence of the depth first walk) The sequence of processes

$$
X_{n}(t)=\frac{V_{n}((2 n-2) t)}{\sqrt{n}} \quad(0 \leq t \leq 1)
$$

converges polynomially to $X=\frac{2}{\sigma} e$.

This theorem allows to derive convergence of moments for some functionals of simple trees:

Let $H(\tau)$ denote the height of $\tau$ (that is the maximum distance between a node and the root) and $P L(\tau)$ the path length of $\tau$ (that is the sum of the distance of each node to the root). Then it is well known that

$$
\frac{H(\tau)}{\sqrt{n}}=\max _{0 \leq t \leq 1} \frac{V_{n}((2 n-2) t)}{\sqrt{n}}
$$

and

$$
\frac{P L(\tau)}{n^{3 / 2}}=\frac{1}{2}\left(n^{-1 / 2}+\left(2-\frac{2}{n}\right) \int_{0}^{1} \frac{V_{n}((2 n-2) t)}{\sqrt{n}} d t\right)
$$

Since $F=\max$ and $F=\int_{0}^{1} . d t$ satisfy $(2)$ it follows that

$$
\mathbf{E}\left(\left(\frac{H_{n}}{\sqrt{n}}\right)^{k}\right) \rightarrow \mathbf{E}\left(\max _{t} e^{k}(t)\right)
$$

and

$$
\mathbf{E}\left(\left(\frac{P L_{n}}{n^{3 / 2}}\right)^{k}\right) \rightarrow \mathbf{E}\left(\int_{0}^{1} \frac{2}{\sigma} e(t) d t\right)^{k}
$$

for any $k>0$.

Remark 5 The limit moments of the height of size $n$ simply generated trees were computed by Flajolet and Odlyzko (1982) (with some error terms, see also De Bruijn, Knuth and Rice, 1972). They already observed 
that the limiting moments coincide with those of the maximum of the Brownian excursion (after renormalization). Thus, as a by-product, they have proved that the height of simply trees, suitably normalized, converges weakly to the maximum of the Brownian excursion.

Aldous (1991), using probabilistic methods, proves that the depth first path associated to size $n$ simple trees, (suitably normalized) converges to the Brownian excursion. Hence, as a corollary he also obtains that the height of simple trees converges weakly to the maximum of the Brownian excursion. The work of Aldous gives a nice explanation of the convergence of the height of simple trees to the maximum of the Brownian excursion, but the obtained convergence is just weak convergence: one cannot deduce from Aldous' results the moments convergence of Flajolet and Odlyzko (1982).

\section{References}

[1] D. Aldous (1991), The continuum random tree. II: An overview., Stochastic analysis, Proc. Symp., Durham/UK 1990, Lond. Math. Soc. Lect. Note Ser. 167, 23-70.

[2] P. Billingsley (1968), Convergence of Probability Measures, John Wiley \& Sons, New York,.

[3] P. Billingsley (1995), Probability and Measure, 3rd ed. Chichester, John Wiley \& Sons.

[4] N.G. de Bruijn, D.E. Knuth, and S.O. Rice (1972), The average height of planted plane trees, Graph Theory Comput., 15-22.

[5] P. Chassaing and J.F. Marckert (2001), Parking functions, empirical processes, and the width of rooted labeled trees, Electron. J. Combin. 8, no. 1.

[6] M. Csörgő and L. Horváth (1993), Weighted Approximations in Probability and Statistics, Wiley Series in Prob. and Math. Stat., Chichester,.

[7] M. Drmota (1994), The height distribution of leaves in rooted trees, Discrete Mathematics and Applications 4, 45-58.

[8] M. Drmota and G. Gittenberger (2004), The width of Galton-Watson trees, Disc. Math. Theoret. Comput. Sci. 6, no. 2, 387-400.

[9] P. Flajolet and A. Odlyzko (1982), The average height of binary trees and other simple trees, J. Comput. System Sci. 25, no. 2, 171-213.

[10] D.Kh. Fuk and S.V. Nagaev (1971), Probability inequalities for sums of independent random variables, Theory Probab. Appl. 16, 643-660.

[11] B. Gittenberger (1999), On the contour of random trees, SIAM J. Discrete Math. 12, 434-458.

[12] J.F. Marckert and A. Mokkadem (2003), The depth first processes of Galton-Watson trees converge to the same Brownian excursion, Ann. Probab 31, no. 3, 1655-1678.

[13] J. Spencer (1997), Enumerating graphs and Brownian motion. Commun. Pure Appl. Math. 50, no. 3, $291-294$.

[14] D. Revuz and M. Yor (1999), Continuous Martingales and Brownian Motion, 3rd ed., Springer, Berlin. 